\title{
Results and Outcomes of Radical Prostatectomy for Low- Risk Prostate Cancer in North African Ethnic Group
}

\author{
Nabil Louardi a, b, Abdelatif Janane ${ }^{\mathrm{a}}$, Abdessamad Elbahria ${ }^{\mathrm{a}}$, Mohammed Ghadouane ${ }^{\mathrm{a}}$, \\ Ahmed Ameur ${ }^{\mathrm{a}}$, Mohammed Abbar ${ }^{\mathrm{a}}$
}

\begin{abstract}
Background: Prostate cancer ( $\mathrm{PCa}$ ) is a leading cause of cancer death in the world. Indeed, its incidence is increasing with diagnoses made increasingly early thanks to the introduction of screening by prostate-specific antigen (PSA). This detection is done with most often localized stages, causes over diagnosis whose main consequence is overtreatment of the low-risk cancers that would have evolved very slowly and not aggressively without any treatment.
\end{abstract}

Methods: To evaluate the pattern of treatment decisions and oncological outcomes among men aged $\geq 60$ and $\geq 70$ years with low-risk $\mathrm{PCa}$ in North African ethnic group, we examined the proportion and outcomes of men with low-risk disease treated with radical prostatectomy (RP) at our institution in the last decade.

Results: Median age of the 166 men in the study cohort was 66 years. Mean serum PSA at diagnosis was $5.9 \mathrm{ng} / \mathrm{mL}$ with an average ratio of $13.77 \%$. At diagnosis, $70.3 \%$ of our patients were symptomatic with lower urinary tract symptom (LUTS) with a suspicious digital rectal examination in $9.7 \%$ of cases. Clinical stage was $\mathrm{T} 1 \mathrm{a} / \mathrm{b}$ in $5.1 \%$, T1c in $79.6 \%$ and T2a in $15.3 \%$ of the patients. All men had Gleason score (GS) 6 PCa on biopsy and all men were treated with open radical retropubic prostatectomy. Except for age, there was no difference in the clinical features of men aged $65-69$ and $\geq 70$ years. One hundred percent of cancers are adenocarcinomas. Final pathological review revealed organ-confined disease in $77.1 \%$ of the men, extracapsular extension (ECE) in $22 \%$, seminal vesicle invasion (SVI) in $8.6 \%$ and lymph node involvement in $3.2 \%$.

Conclusion: The challenge lies in identifying the aggressiveness of the cancer at diagnosis, and the ability to predict the individual risk of progression, active surveillance (AS) strategy needs to be validated by long-term results, new therapy options are currently being evalu-

Manuscript accepted for publication October 23, 2015

aDepartment of Urology, Military Hospital of Instruction Mohammed V, Rabat, Morocco

${ }^{b}$ Corresponding Author: Nabil Louardi, Department of Urology, Military Hospital of Instruction Mohammed V, Rabat, Morocco.

Email: n.louardi@hotmail.fr

doi: http://dx.doi.org/10.14740/wjnu241w ated, and we consider that RP is an adequate therapy in men with low risk of d'Amico features.

Keywords: Prostate cancer; Radical prostatectomy; Low risk; North African; Ethnic group

\section{Introduction}

Low-risk tumors are conventionally defined by the d'Amico classification. The use of multiparametric magnetic resonance imaging (MRI) helps to better characterize these tumors. Prostate cancer $(\mathrm{PCa})$ management decisions depend on disease stage and grade, among other prognostic factors. Men with clinically low-risk disease are often well suited for active surveillance (AS) rather than immediate treatment. However, despite improvements in clinical and pathologic assessment, there are considerable levels of upgrading and upstaging between biopsy and radical prostatectomy (RP).

In men with localized $\mathrm{PCa}$ and a life expectancy $>10$ years, the goal of RP by any approach must be eradication of disease, while preserving continence and whenever possible potency [1]. There is no age threshold for RP and a patient should not deny this procedure on the grounds of age alone [2]. Increasing comorbidity greatly increases the risk of dying from non-PCa-related causes [3]. An estimation of life expectancy is paramount in counseling a patient about surgery [4].

Today, the contribution of pathology, molecular biology and imaging can reveal in the low-risk group, the notion of tumors at very low risk of progression. Patients with such tumors are the best candidates for AS.

To evaluate the pattern of treatment decisions and oncological outcomes among men aged $\geq 60$ and $\geq 70$ years with low-risk $\mathrm{PCa}$, we examined the proportion and outcomes of men with low-risk disease treated with RP at our institution in the last decade.

\section{Materiel and Methods}

All men that participated in the Prostate Cancer Research were initially diagnosed with low-risk PCa (clinical stage $<\mathrm{T} 2 \mathrm{a}$, prostate-specific antigen $(\mathrm{PSA})<10 \mathrm{ng} / \mathrm{mL}$, one or two posi-

Articles @ The authors | Journal compilation @ World J Nephrol Urol and Elmer Press Inc ${ }^{\mathrm{TM}} \quad$ | www.wjnu.elmerpress.com

This is an open-access article distributed under the terms of the Creative Commons Attribution License, which permits unrestricted use, distribution, and reproduction in any medium, provided the original work is properly cited 
tive biopsy cores, Gleason score (GS) $<6$, and prostate-specific antigen velocity (PSAV) $<0.75 \mathrm{ng} / \mathrm{mL} /$ year) and underwent RP between February 2001 and July 2014.

We have evaluated on a retrospective way the proportion and outcomes of men aged $\geq 60$ years with low-risk disease who underwent RP at our institution.

Sub-analysis was done on men aged $\geq 70$ years to determine whether outcomes among older men differed by age.

Independent variables at diagnosis were age, PSA value, PSAV, clinical TNM stage, biopsy Gleason grade, biopsy cores sampled and positive, and percentage of tumor tissue.

More than 190 patients were queried for men aged $\geq 60$ years with low-risk PCa. Pathological and survival outcomes were assessed. Twenty-four patients without complete data on preoperative PSA, clinical stage and biopsy Gleason sum were excluded from analysis.

Our study involved 166 cases. Clinical, biological, histological and evolving of these patients were analyzed. A comparative analysis of our results was made with data from a review of contemporary literature on the management of PCa.

\section{Results}

Median age of the 166 men in the study cohort was 66 years (range 63 - 75), including 65 (range 63 - 69) in 154 and 71 (range 70 - 75) in $12(\mathrm{P}<0.005)$. Mean serum PSA at diagnosis was $5.9 \mathrm{ng} / \mathrm{mL}$ (range 0.2 - 10), including $5.3(0.2$ - 10) and 5.7 $(0.2-10)$ in those aged $60-69$ and $\geq 70$ years, respectively, with an average ratio of $13.77 \%$.

At diagnosis, $70.3 \%$ of our patients were symptomatic with lower urinary tract symptom (LUTS) with a suspicious digital rectal examination in $9.7 \%$ of cases. Clinical stage was $\mathrm{T} 1 \mathrm{a} / \mathrm{b}$ in $5.1 \%$, T $1 \mathrm{c}$ in $79.6 \%$ and $\mathrm{T} 2 \mathrm{a}$ in $15.3 \%$ of the patients. All men had GS 6 PCa on biopsy. All men were treated with open radical retropubic prostatectomy associated with bilateral ilio-obturator lymph node dissection in $37.9 \%$ of cases. During surgery, we had 10 cases $(6.02 \%)$ with bleeding episodes requiring transfusion, and immediate and early postoperative; 12 cases $(7.22 \%)$ with surgical site infection, and three cases $(1.87 \%)$ with localized lymphorrhea. Except for age, there was no difference in the clinical features of men aged $60-69$ and $\geq 70$ years (Table 1 ).

Of the 166 patients, the diagnosis was made by prostate biopsy in 155 patients and 11 patients after transurethral resection of the prostate (TURP). The number of positive cores ranged from 1 to 2 . One hundred percent of cancers are adenocarcinomas.

\section{Pathological features}

Final pathological review revealed organ-confined disease in $77.1 \%$ of the men, extracapsular extension (ECE) in $22 \%$, seminal vesicle invasion (SVI) in $8.6 \%$ and lymph node involvement in $3.2 \%$. After RP, 63 men $(38.3 \%)$ had unfavorable pathological features. There was no difference in pathological characteristics between men aged $60-69$ and $\geq 70$ years.
Table 1. Clinical and Biological Data of the Patients

\begin{tabular}{|ll}
\hline Features of men & $\begin{array}{l}\text { Number of patients } \\
\text { (percentage) }\end{array}$ \\
\hline Age (years) & \\
\hline Average: 60 years (range $63-75)$ & \\
$\quad 60$ - 69 & $154(92.77 \%)$ \\
$>70$ & $12(7.22 \%)$
\end{tabular}

After surgery, overall upgrade was $43 \%$ and did not differ significantly between groups. Most (57\%) patients had Gleason grade $6(3+3), 37 \%$ had grade $3+4$, and $6 \%$ had grade 4 +3 or higher. The upstage rate was $9 \%$.

\section{Survival outcomes}

At a median follow-up of 5 years, eight patients (4.2\%) experienced biochemical recurrence (BCR) and 21 (11\%) had died, of whom one $(0.5 \%)$ died of PCa and three $(1.5 \%)$ died of cardiovascular disease. The man died of PCa at a median of 9 years postoperatively. For the entire cohort, actuarial 5- and 10 -year BCR-free survival was $93.2 \%$ and $89.2 \%$, overall survival (OS) was $96.1 \%$ and $83.5 \%$, and $\mathrm{PCa}$-specific survival was $99.7 \%$ and $98.4 \%$, respectively. For men aged $60-69$ years, actuarial 5- and 10-year BCR-free survival was 93.8\% and $90.1 \%$, OS was $96.8 \%$ and $84.6 \%$, and PCa-specific survival was $99.9 \%$ and $98.9 \%$ respectively.

For men aged $\geq 70$ years, actuarial 5- and 10-year BCRfree survival was $88 \%$ and $79.3 \%$, OS was $88.4 \%$ and $70.1 \%$, and PCa-specific survival was $98.4 \%$ and $91.8 \%$, respectively.

There was no difference in BCR-free or PCa-specific survival when comparing men aged $60-69$ and $\geq 70$ years $(\mathrm{P}>$ 0.07 and $\mathrm{P}>0.07$, respectively). Compared to men aged 60 - 69 years, those aged $\geq 70$ years had lower OS and cardiovascular specific survival (each $\mathrm{P}<0.005$ ).

According to post-therapeutic prostate-specific antigen doubling time (PSADT) and detailed characteristic and pa- 
rameters of specimen histology, we have performed radio or systemic treatment adjuvant to radical surgery: $5 \%$ of patients received adjuvant radiotherapy treatment and $8 \%$ of patients received adjuvant hormone therapy.

\section{Discussion}

Currently, $40-50 \%$ of PCa diagnosed in Europe belong to the group at low risk of d'Amico. In literature review, Ploussard reported the results of the external validation Epstein criteria. The risk of undergrading and understaging, in other words, the risk of missing an aggressive disease, was close to $30 \%[5,6]$.

Part of the low-risk PCa is associated with a moderate risk of metastatic progression or no. It is therefore legitimate to ask whether these tumors should be treated consistently. Two types of studies can answer this question: longitudinal studies of patients under surveillance (uninformative because of their too short follow-up), and studies comparing monitoring to a conventional total treatment. Both types of studies include several comparative studies. Three populations bring Scandinavian reply. The study elements reported by Bill-Axelson et al [7] included 695 patients with a "localized" cancer between 1989 and 1999. Patients were randomized, and then treated with either RP or by watchful waiting (WW). After a follow-up of 18 years, progression-free survival and specific survival were better in the group of operated patients. These results are difficult to apply today. Indeed, there were few low-risk tumors ( $12 \%$ of non-palpable tumors, PSA average $12 \mathrm{ng} / \mathrm{mL}$ ), and the management arrangements in the group of monitored patients (hormonal treatment for clinical progression) are not those of AS. A subgroup analysis was performed, showing that the RP did not provide significant benefit after age 70 years [8]. For younger patients, oncological benefit is definite for stage T2 tumors and GS $\geq 7$, and possible for two types of tumors stage T2 and GS 6 or T1 stage and GS 7. However, it seemed nil for stage T1 tumors and GS 6 . The reduction in mortality at 10 years depended on the characteristics of cancer: clear $(17.2 \%)$ for high-risk tumors, but limited (4.5\%) for low-risk tumors. A retrospective study comparing the watchful treatment immediately (RP or radiotherapy) was published from the US databases Medicare [9]. It included 44,630 patients, aged $65-80$ years with clinically localized PCa. This study showed, with followup of 12 years, significant improvement in OS in the group of patients treated immediately. This study is very heterogeneous, with a high proportion of tumors T2 and/or GS 7 and included many older men. It does not conclude the feasibility of AS for low-risk tumors, especially in young people. The trial PIVOT recently published also attempted to answer this question [10]. In this study, 731 patients with clinically localized cancer were randomized (1994 - 2002) into two groups: RP or watchful. With a median follow-up of 10 years, OS and specific survival were similar in both groups. The respective rates of overall and specific mortality were $49.9 \%$ and $8.4 \%$ in the monitoring group, versus $47 \%$ and $5.8 \%$ in the prostatectomy group (not significant). If we take into account only patients with a PSA $>$ $10 \mathrm{ng} / \mathrm{mL}$, specific mortality was significantly increased $7.2 \%$ in the monitoring group and $13.2 \%$ overall mortality. This study suggests that for low-risk tumors, prostatectomy did not significantly reduce mortality. It does reduce mortality than for intermediate or high risk of tumors. However, this study raises at least three problems. On the one hand, its workforce is reduced: of 5,023 eligible patients, only 731 (14.5\%) agreed to randomization; on the other hand, contamination monitoring arm was significant: about $20 \%$ of patients were actually treated. It is logical to think that these patients had the most aggressive tumors, which may have affected the results. Most importantly, the occurrence of metastases was significantly more frequent in the monitoring group (10.6\% versus $4.7 \%)$, raising fears of an increased risk of mortality over long term. The PIVOT study therefore does not have enough power to allow a definitive conclusion [11].

AS is a curative treatment option moving the time of treatment while still in the curability window. It was established during the past decade in the treatment of PCa strategy with a goal of reducing the rate of treatment in patients with localized PCa at very low risk without abandoning the idea of a radical treatment $[7,9]$. Recently, the only available data on AS are from non-mature randomized studies with a follow-up under 10 years. However, AS should be offered to highly selected patients because of the risk of cancer progression. This idea of hyper patient selection is also supported by other studies such as Johansson who showed that there was a high risk of cancer death in patients with a life expectancy of over 15 years with tumors well and moderately differentiated [9]. In light of these data, it is essential that the selection of patients for AS is well pointed.

In our series, no patient had AS. Referring to the different selection criteria, 15 patients met the criteria and among the 15 , three patients had pT0 which gives us a sense of overtreatment for these patients. Maybe we should properly evaluate these patients and talk to them for a possible AS.

The role of PSADT to identify the need for intervention was recently challenged [10]. In a cohort of 290 men who underwent AS for low-risk PCa, 35\% developed biopsy progression (Gleason score $\geq 7$, more than two positive cores, or $>$ $50 \%$ core involvement). PSADT was not significantly associated with biopsy progression $(\mathrm{P}=0.83)$, nor was PSAV $(\mathrm{P}=$ $0.06)$. In another study, $36 \%$ of men under AS demonstrated disease progression on repeat biopsy [11]. The 5-year progression-free probability was $82 \%$ for patients with a negative first repeat biopsy compared with $50 \%$ for patients with a positive repeat biopsy. Both trials underline the need for annual surveillance repeat biopsies to monitor men adequately under AS independent of the results of PSADT.

RP is the only treatment for localized PCa that has shown a cancer-specific survival benefit when compared with WW in a prospective randomized trial $[12,13]$ and most of the patients recruited were of intermediate risk and did not harbor screendetected $\mathrm{PCa}$, so these data cannot be automatically transferred into daily routine practice. Nerve-sparing RP represents the approach of choice in all men with a normal erectile function and organ-confined disease. The need for and the extent of pelvic lymphadenectomy is controversial. The risk of lymph node involvement is low in men with low-risk $\mathrm{PCa}$ and $<50 \%$ positive biopsy cores [14].

Neoadjuvant androgen deprivation does not provide a significant advantage in OS and progression-free survival and 
therefore has no role in the surgical treatment of $\mathrm{PCa}$ [15].

The decision to offer RP in cases of low-risk cancer should be based upon the probabilities of clinical progression, side effects and potential benefit to survival [16]. It might therefore be reasonable to propose AS to selected patients whose tumors are most likely to be insignificant. Apart from disease characteristics, age, comorbidities and individual patient preferences impact the choice for surgery vs. AS and should be considered in shared decision making. A recent study assessed the effect of age, health status and patient preferences on outcomes of surgery vs. AS for low-risk PCa. As expected, older age and worse baseline health status were associated with a smaller benefit in PCa-specific mortality and life expectancy with surgery, and increased incremental years with treatment side effects [17].

Pelvic lymph node dissection is not necessary in low-risk PCa because the risk for positive lymph nodes does not exceed $5 \%[18]$.

Having the percentage of reaching capsular, reaching vesicular and GS of $7(3+4)$ and $7(4+3)$ in specimen of RP, otherwise, when we explain to our patients accuracy of the different nomograms and their results in upstaging and upgrading, the majority of them choose surgery option.

\section{Conclusion}

The challenge lies in identifying the aggressiveness of the cancer at diagnosis, and the ability to predict the individual risk of progression, and AS strategy needs to be validated by longterm results. New therapy options are currently being evaluated. The conventional parameters (d'Amico) are insufficient to date. The MRI's contribution to better characterize tumors and progress of molecular biology should help predict the risk of progression of each lesion, and help in choosing a treatment because of considerable levels of upgrading and upstaging between biopsy and RP, and we consider that RP is an adequate therapy in men with low risk of d'Amico features.

\section{Abbreviations}

MRI: magnetic resonance imaging; AS: active surveillance; RP: radical prostatectomy; PCa: prostate cancer; PSA: prostate-specific antigen; LUTS: lower urinary tract symptom; TURP: transurethral resection of the prostate; ECE: extracapsular extension; PSAV: prostate-specific antigen velocity; SVI: seminal vesicle invasion; BCR: biochemical recurrence; OS: overall survival; PSADT: prostate-specific antigen doubling time; WW: watchful waiting; GS: Gleason score

\section{References}

1. Albertsen PC, Moore DF, Shih W, Lin Y, Li H, Lu-Yao GL. Impact of comorbidity on survival among men with localized prostate cancer. J Clin Oncol. 2011;29(10):13351341.

2. Droz JP, Balducci L, Bolla M, Emberton M, Fitzpatrick JM, Joniau S, Kattan MW, et al. Background for the pro- posal of SIOG guidelines for the management of prostate cancer in senior adults. Crit Rev Oncol Hematol. 2010;73(1):68-91.

3. Bianco FJ, Jr., Scardino PT, Eastham JA. Radical prostatectomy: long-term cancer control and recovery of sexual and urinary function ("trifecta"). Urology. 2005;66(5 Suppl):83-94.

4. Walz J, Gallina A, Saad F, Montorsi F, Perrotte P, Shariat $\mathrm{SF}$, Jeldres $\mathrm{C}$, et al. A nomogram predicting 10year life expectancy in candidates for radical prostatectomy or radiotherapy for prostate cancer. J Clin Oncol. 2007;25(24):3576-3581.

5. Ploussard G, Azancot V, Nicolaiew N, Xylinas E, Salomon L, Allory Y, Vordos D, et al. The effect of prostatespecific antigen screening during the last decade: development of clinicopathological variables independently of the biopsy core number. BJU Int. 2010;106(9):12931297.

6. Ploussard G, Epstein JI, Montironi R, Carroll PR, Wirth M, Grimm MO, Bjartell AS, et al. The contemporary concept of significant versus insignificant prostate cancer. Eur Urol. 2011;60(2):291-303.

7. Bill-Axelson A, Holmberg L, Ruutu M, Garmo H, Stark JR, Busch C, Nordling S, et al. Radical prostatectomy versus watchful waiting in early prostate cancer. N Engl J Med. 2011;364(18):1708-1717.

8. Vickers A, Bennette C, Steineck G, Adami HO, Johansson JE, Bill-Axelson A, Palmgren J, et al. Individualized estimation of the benefit of radical prostatectomy from the Scandinavian Prostate Cancer Group randomized trial. Eur Urol. 2012;62(2):204-209.

9. Wong YN, Mitra N, Hudes G, Localio R, Schwartz JS, Wan F, Montagnet C, et al. Survival associated with treatment vs observation of localized prostate cancer in elderly men. JAMA. 2006;296(22):2683-2693.

10. Al Otaibi M, Ross P, Fahmy N, Jeyaganth S, Trottier H, Sircar K, Begin LR, et al. Role of repeated biopsy of the prostate in predicting disease progression in patients with prostate cancer on active surveillance. Cancer. 2008;113(2):286-292.

11. Ross AE, Loeb S, Landis P, Partin AW, Epstein JI, Kettermann A, Feng Z, et al. Prostate-specific antigen kinetics during follow-up are an unreliable trigger for intervention in a prostate cancer surveillance program. J Clin Oncol. 2010;28(17):2810-2816.

12. Bill-Axelson A, Holmberg L, Ruutu M, Haggman M, Andersson SO, Bratell S, Spangberg A, et al. Radical prostatectomy versus watchful waiting in early prostate cancer. N Engl J Med. 2005;352(19):1977-1984.

13. Bill-Axelson A, Holmberg L, Filen F, Ruutu M, Garmo $\mathrm{H}$, Busch C, Nordling S, et al. Radical prostatectomy versus watchful waiting in localized prostate cancer: the Scandinavian prostate cancer group- 4 randomized trial. J Natl Cancer Inst. 2008;100(16):1144-1154.

14. Heidenreich A, Pfister D, Thuer D, Brehmer B. Percentage of positive biopsies predicts lymph node involvement in men with low-risk prostate cancer undergoing radical prostatectomy and extended pelvic lymphadenectomy. BJU Int. 2011;107(2):220-225. 
15. Shelley MD, Kumar S, Wilt T, Staffurth J, Coles B, Mason MD. A systematic review and meta-analysis of randomised trials of neo-adjuvant hormone therapy for localised and locally advanced prostate carcinoma. Cancer Treat Rev. 2009;35(1):9-17.

16. Kattan MW, Eastham J. Algorithms for prostate-specific antigen recurrence after treatment of localized prostate cancer. Clin Prostate Cancer. 2003;1(4):221-226.

17. Liu D, Lehmann HP, Frick KD, Carter HB. Active sur- veillance versus surgery for low risk prostate cancer: a clinical decision analysis. J Urol. 2012;187(4):12411246.

18. Briganti A, Larcher A, Abdollah F, Capitanio U, Gallina A, Suardi N, Bianchi M, et al. Updated nomogram predicting lymph node invasion in patients with prostate cancer undergoing extended pelvic lymph node dissection: the essential importance of percentage of positive cores. Eur Urol. 2012;61(3):480-487. 